\title{
Research on coordinated controller design method based on fault early warning model
}

\author{
Xu Jian ${ }^{1}$, Jia Shaokun ${ }^{1}$, Bai Jie ${ }^{1}$, Zhao Kunpeng $^{2}$, Wu Minglei ${ }^{3}$, Ding Qiang ${ }^{4}$ \\ ${ }^{1}$ Beijing Fibrlink Communications Co, Ltd, Tianjin, 300040, China \\ ${ }^{2}$ Customer service center of State Grid Corporation of China, Tianjin, 300300, China \\ ${ }^{3}$ State Grid Tianjin integrated energy service Co.LTD, Tianjin, 300456, China \\ ${ }^{4}$ State Grid Jiangsu integrated energy service Co.LTD, Jiangsu, 210019, China
}

\begin{abstract}
With increasing degree of power electronics in power system, the interaction between controllers of different FACTS devices poses a greater threat to security and stability of the system. In this paper, a linearized state space model with SVC and STATCOM was established, and the negative interaction mechanism of FACTS device in transient process was analyzed and summarized considering control characteristics, operation mode and setting principle of PI controllers. A model predictive control (MPC) is proposed to replace traditional PI link to establish a coordination controller to overcome the difficulties of PI control parameter setting and global optimization.
\end{abstract}

\section{Introduction}

Flexible AC transmission system(FACTS) equipment is a new type of power equipment which integrates modern power electronic technology, modern communication technology and modern control theory. it is an important means[1] to enhance the flexibility and controllability of transmission system, enhance the transient stability and transmission capacity of power network and suppress system oscillation.

There are three kinds of coordinated control methods for FACTS devices: linear coordinated control method, nonlinear coordinated control method and intelligent algorithm coordinated control method[2]. Literature[3] consider the coordination and control of SVC and TCSC in multi-machine systems, an improved fuzzy advanced lag coordination controller is proposed, and the ACACO is used to optimize the parameters of fuzzy control, the simulation results show that the scheme has good dynamic performance. Document[4] use wavelet transform to analyze the necessity of coordinated configuration, using the improved multi-objective quantum genetic algorithm and limit learning machine, The effectiveness of the algorithm is verified in SVC and TCSC system simulation. The paper[5] that the problem of coordinated control between UPFC controllers is transformed into the problem of parameter tuning, And establish the multiobjective optimization model of parameter optimization, a MOPSO is used to coordinate and optimize the control parameters.

To sum up, the current coordinated control method is still based on the traditional PI control. The main optimization ideas are as follows: using the control theory to optimize the configuration of the system or optimizing the control gain through the intelligent algorithm. Has the following shortcomings :1) the intelligent algorithm modeling is complex and the portability is poor 2) is restricted by the diversity of FACTS devices 3 ) the system model only considers the steady state operation.

\section{Power System Model with SVC and STATCOM}

\subsection{Power System Dynamic Model with SVC and STATCOM}

SVC and STATCOM devices are added to the single machine infinity model in literature [6] to obtain the research model shown in figure 1 . The SVC is installed at the node $\mathrm{Vt}$ to improve the damping characteristics and voltage stability, and the STATCOM installation at the node $\mathrm{Vc}$ can effectively improve the transient performance of the voltage, and it is also of great significance to stabilize the power angle of the generator.

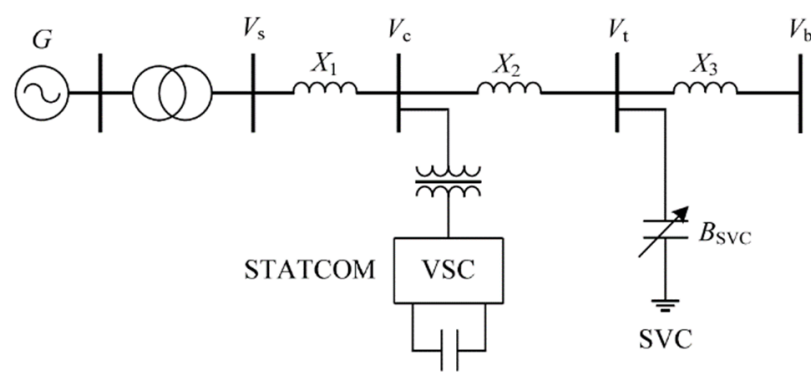

Fig. 1 SIMB Power system with SVC and STATCOM

Among them, the exciter and synchronous generator can be described by the third order model, and the dynamic equation is

\footnotetext{
* Corresponding author: HBBDndyz@163.com
} 


$$
\left\{\begin{array}{l}
\dot{\delta}=\omega_{0} \omega \\
\dot{\omega}=\frac{P_{m}-P_{e}-D \omega}{M} \\
\dot{E_{q}^{\prime}}=\frac{-\left(E_{q}-E_{f d}\right)}{T_{d 0}^{\prime}} \\
E_{f d}^{\prime}=-\frac{1}{T_{A}} E_{f d}+\frac{K_{A}}{T_{A}}\left(V_{s 0}-V_{s}\right)
\end{array}\right.
$$

Formula: $\delta, \omega, D, M$ are generator work angle, angular velocity, damping coefficient and inertia time constant, $E_{q}, E_{f d}$ no-load electromotive force, excitation electromotive force; other parameters and variables meaning reference[7].

The SVC is usually composed of a fixed capacitor fixed capacitor(FC) and a thyristor control reactor thyristor controlled reactor(TCR) in parallel. By controlling the conduction angle of the thyristor $\alpha$ the equivalent reactance of the on-in circuit is changed, and then the equivalent capacitance of the SVC is changed. Since the SVC voltage and stability control are both pure gain control, simplification can be achieved:

$$
\left.\Delta B_{T C R}=\left(-B_{T C R}+B_{T C R 0}+\Delta V_{t}\right) / T_{S}\right)
$$

Formula: $B_{T C R}$ inductance branch admittance; $B_{\text {TCR0 }}$ is the initial admittance of inductance branch; $T_{S}$ is the SVC controller time constant.

The STATCOM is usually composed of a three-phase voltage inverter (VSC) and a DC capacitor based on gateoff thyristor. By changing the parameters of the inverter V0 a controllable voltage source is generated. Then the purpose of controlling the power flow and voltage stability of the system is achieved. According to its operating characteristics, the [12] of its dynamic equation is

$$
\left\{\begin{array}{l}
\bar{I}_{1 o}=I_{1 o d}+j I_{1 o q} \\
\bar{V}_{o}=C V_{D C}(\cos \varphi+j \sin \varphi)=C V_{D C} \angle \varphi \\
\frac{d V_{D C}}{d t}=\frac{C}{C_{D C}}\left(I_{1 o d} \cos \varphi+I_{1 o q} \sin \varphi\right)
\end{array}\right.
$$

Formula : $I_{1 o}, V_{o}$ and $V_{D C}$ are the injection current, compensation voltage and DC voltage respectively; $C$ is the relevant parameters of the modem ; $C=m k, k$ is the ratio of $\mathrm{AC} / \mathrm{DC}$ voltage of the inverter, which is related to the structure of the inverter.

The equations of state of a single machine with infinite SVC and STATCOM can be obtained by combining the two dynamic models and linearization:

$$
\left\{\begin{array}{l}
x=A x+B u \\
y=C x+D u
\end{array}\right.
$$

$$
\begin{aligned}
& \text { Formula : } x=\left[\begin{array}{lllll}
\Delta \delta & \Delta \omega & \Delta E_{q}^{\prime} & \Delta E_{f d} & \Delta V_{D C}
\end{array}\right]^{T} \text { is } \\
& \text { the state variable and } u=\left[\begin{array}{lll}
\Delta m & \Delta \varphi & \Delta \alpha
\end{array}\right]^{T} \text { is the } \\
& \text { control input variable of the device and } \\
& y=\left[\begin{array}{lll}
\Delta V_{c} & \Delta V_{D C} & \Delta V_{t}
\end{array}\right]^{T} \text { is the system output variable. }
\end{aligned}
$$

\subsection{SVC and STATCOM interaction analysis}

The controller of the FACTS device connected in parallel in the system usually only considers the application scene of a single device and designs the corresponding PI controller. Although this method is very convenient and effective for the design of a single device, with the investment of a large number of FACTS devices and the diversification of the application scenarios of the device itself, the design of a single device one by one can no longer meet the current requirements. At the same time, the traditional design method does not consider the coupling of control variables, which leads to the performance of PI controller greatly weakened or even contradictory.

For the power system shown in figure 1, Literature [8] using the relative gain matrix (RGA) method to give a quantitative analysis of the interaction between the two, Noting that the interaction between SVC and STATCOM is related to the electrical distance between devices, The farther the electrical distance, the less the interaction; However, it is affected by the solidification of the grid structure during the actual operation, The electrical distance between devices can not normally change, Therefore, the method is not very practical. literature used effective relative energy matrix (EREA), canonical form (Normal Form) methods to effectively analyze the interaction between other types of FACTS devices. Apparently, FACTS the interaction between the devices, For better resolution of the interaction between FACTS devices, In this paper, a new coordination controller is designed by using model predictive control principle.

\section{Power System Model with SVC and STATCOM}

Model predictive control [9] is a new type of computer control algorithm developed in 1960s, including model algorithm control (MAC), dynamic matrix control (DMC) and generalized predictive control (GPC). Different from other predictive control methods, dynamic matrix control (dynamic matrix control) is widely used.

\section{1.model prediction}

For a linear multivariable system, each output will be affected by multiple input changes, and its dynamic changes can be superimposed by the changes of each input. Therefore, considering the change of output $y_{i}$ caused by input $u_{j}$, there is an increment $\Delta u_{j}(k)$ of 
$u_{j}$ at the point of $k$, and the output prediction value of

$y_{i}$ at the point of $N$ can be obtained:

$$
\hat{y}_{i, N 1}(k)=\hat{y}_{i, N 0}(k)+a_{i j} \Delta u_{j}(k)
$$

Formula:

$$
\begin{gathered}
\hat{y}_{i, N 1}(k)=\left[\hat{y}_{i, 1}(k+1 \mid k) \cdots \hat{y}_{i, 1}(k+N \mid k)\right]^{T} \\
\hat{y}_{i, N 0}(k)=\left[\hat{y}_{i, 0}(k+1 \mid k) \cdots \hat{y}_{i, 0}(k+N \mid k)\right]^{T}
\end{gathered}
$$

Each component of $\hat{y}_{i, N 0}(k)$ represents the initial output prediction value of $y_{i}$ at the point of $N$ in the future when the total control quantity remains unchanged at the point of $k$.

At the moment of $k$, considering the $M$ incremental changes of input $u_{j}$ in turn, the initial prediction value of $y_{i}$ at the $P$ point is obtained as follows :

$$
\hat{y}_{i, P M}(k)=\hat{y}_{i, P 0}(k)+A_{i j} \Delta u_{j, M}(k)
$$

Formula:

$$
\begin{aligned}
& \hat{y}_{i, P M}(k)=\left[\hat{y}_{i, M}(k+1 \mid k) \cdots \hat{y}_{i, M}(k+N \mid k)\right]^{T} \\
& \hat{y}_{i, P 0}(k)=\left[\hat{y}_{i, 0}(k+1 \mid k) \cdots \hat{y}_{i, 0}(k+P \mid k)\right]^{T}
\end{aligned}
$$$$
A_{i j}=\left[\begin{array}{ccc}
a_{i j}(1) & & 0 \\
\vdots & \ddots & \\
a_{i j}(M) & \cdots & a_{i j}(1) \\
\vdots & & \vdots \\
a_{i j}(P) & \cdots & a_{i j}(P-M+1)
\end{array}\right]
$$

$$
\Delta u_{j, M}(k)=\left[\Delta u_{j}(k) \cdots \Delta u_{j}(k+M-1)\right]^{T} . M . P
$$
are the control time domain and optimization time domain, general provisions $M \leq P \leq N . k+i \mid k$ denotes a prediction of $k+i$ at $k$ point. Dynamic matrix $A_{i j}$ of unit step response coefficient $a_{i j}$.

\section{Case}

\subsection{Basic data}

During the steady operation of power system, the grid structure of the system is often invariant and the power flow is stable. The traditional PI controller is usually designed according to the linearization model of the steady state. However, when the transient fault occurs, the system grid structure and power flow change, and the linearization model of the system will change accordingly, which leads to the failure of the traditional PI controller based on the steady-state model to obtain the ideal control performance in the transient process.

therefore, in order to test the practicability and effectiveness of this scheme more realistically, a PSCAD/EMTDC simulation model is established as shown in figure 1, and tested from the point of view of steady state and transient state. The system parameters are shown in Table 1.

Tab. 1 PSCAD/EMDTC Simulation parameters

\begin{tabular}{cccc}
\hline parameter & value & parameter & value \\
\hline $\mathrm{G}$ & $100 \mathrm{MVA}$ & $\mathrm{V}_{\mathrm{G}}$ & $13.8 \mathrm{kV}$ \\
$\mathrm{SVC}$ & $30 \mathrm{Mvar}$ & STATCOM & $\pm 30 \mathrm{Mvar}$ \\
$\mathrm{V}_{\text {base }}$ & $110 \mathrm{kV}$ & $\mathrm{V}_{\mathrm{DC}}$ & $37.5 \mathrm{kV}$ \\
\hline
\end{tabular}

Steady-state performance analysis

The voltage curve of the SVC、STATCOM AC side is shown in Fig .2-3 considering the grid connection of the FACTS device and its stable dynamic condition. We can see from figure 2-3 that when the system is put into operation in the MPC control mode, there is still a certain overshoot under the PI control mode. After $1.2 \mathrm{~s}$, the voltage waveform of the two control modes is kept near 1 $\mathrm{pu}$. The waveform is smoother and no obvious fluctuation, while PI control still has a small fluctuation.

Under steady-state operation MPC and PI control modes can meet the control requirements under steadystate conditions. The advantage of adopting MPC control mode is that the overshoot is much smaller than that of PI control, and the bus voltage is more stable under MPC control in the steady state process. Obviously, when the grid structure of the system is not changed, the control model is in the original working state, and the control of both can meet the control requirements, but the control performance of the MPC is still better than that of the PI control.

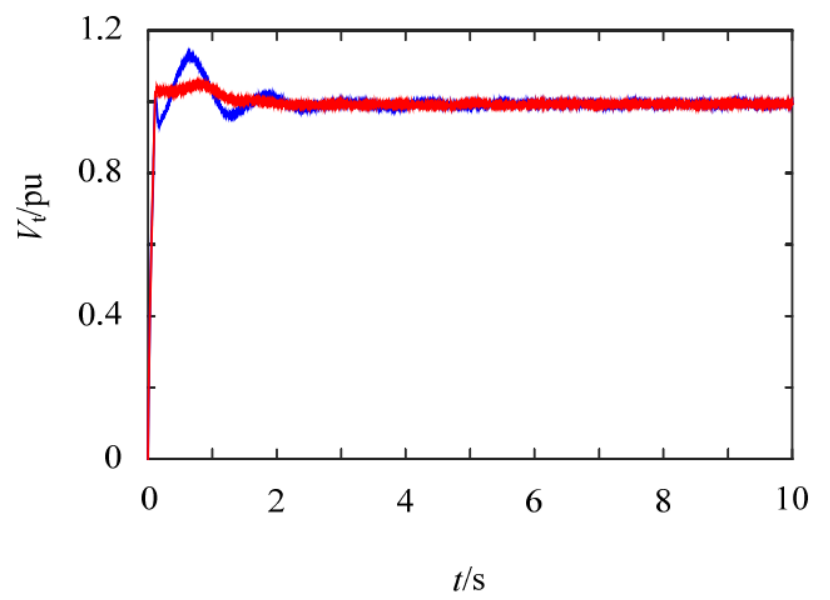

Fig. 2 Steady voltage curve of SVC 


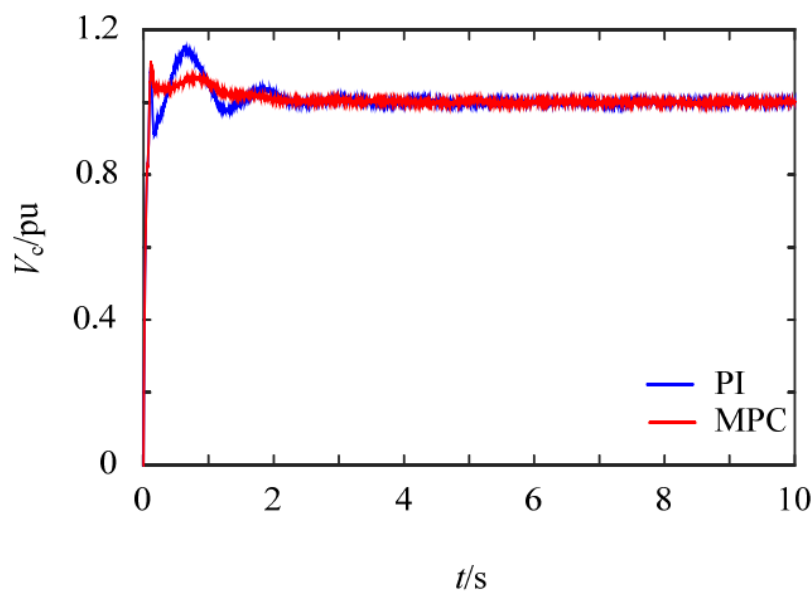

Fig. 3 Steady voltage curve of STATCOM

\section{Conclusion}

Because PI controller can only play an advantage in loop control, it is difficult to meet the control requirements in the face of increasingly complex system environment. In this paper, a method of designing coordination controller based on model predictive control principle is proposed to solve the control problem of nonlinear and strongly coupled multivariable systems. The main conclusions are as follows:

1) The linearized state space model with SVC and STATCOM single machine system is established, and the negative interaction effect under transient process is analyzed by using the step response of the control model. The limitations of the PI controller in the device are revealed when the system grid structure and power flow change.

2) The coordination controller of SVC and STATCOM is designed by using the principle of model predictive control, and the general control structure of the controller is given. Compared with the traditional PI controller, the model predictive controller can better coordinate the interaction between the controllers and effectively overcome the disadvantage that the PI controller can not cope with the change of model parameters.

3) The effectiveness and practicability of the proposed method are verified by PSCAD/EMTDC simulation, which provides a new solution for the design of FACTS controller and the coordinated control of interaction.

\section{References}

1. Zhang Wenliang, Tang Guangfu, Zha Kunpeng, et al. Application of advanced power electronics in smart grid[J]. Proceedings of theCSEE, 2010 , 30(4): 1-7(in Chinese).

2. Gao Lei, Chu Xiaojie, Tang Yong, et al. Reviews on interactions andcoordinated control among multiple FACTS[J] . Power System Technology , $2016,40(12): 3749-3755$ (in Chinese).
3. Lu C F, Hsu C H, Juang C F. Coordinated control of flexible $\mathrm{AC}$ transmission system devices using an evolutionary fuzzy lead-lag controller with advanced continuous ant colony optimization[J] . IEEE Transactions on Power Systems, 2013, 28(1): 385-392.

4. Huang Liuqiang, Guo Jianbo, Sun Huadong, et al . Intelligent computation based coordinated configuration of multi-facts devices [J] . Power System Technology, 2013, 37(4) : 942-946(in Chinese).

5. Yan Nannan, Fu Zhengcai. Coordinated design of UPFC based on multi-objective particle swarm optimization[J] . Power System Protection and Control, 2010(8): 43-48(in Chinese).

6. Zhang $\mathrm{L}$, Zhang $\mathrm{P} \mathrm{X}$, Wang $\mathrm{H} \mathrm{F}$, et al. Interaction assessment of FACTS control by RGA for the effective design of FACTS damping controllers $[\mathrm{J}]$. IEE Proceedings-Generation , Transmission and Distribution, 2006, 153(5): 610-616.

7. Kundur P, Balu N J, Lauby M G. Power system stability and control[M]. New York: McGraw-hill, 1994: 728-732.

8. Jiang Quanyuan, Zou Zhenyu, Wu Hao, et al. Interaction analysis of FACTS controllers based on RGA principle[J]. Proceedings of the CSEE, 2005, 25(11): 23-28(in Chinese).

9. Qin S J, Badgwell T A. A survey of industrial model predictive control technology[J] . Control engineering practice, 2003, 11(7): 733-764. 